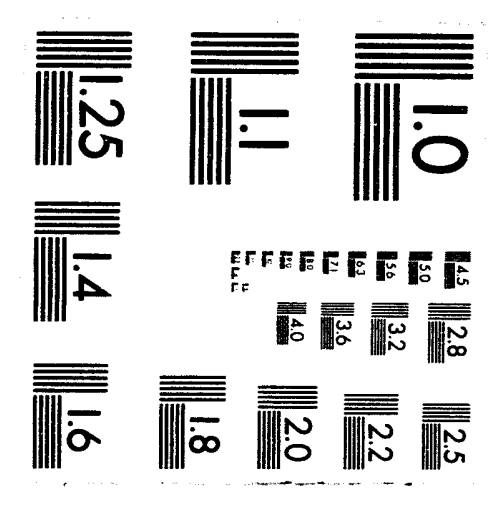




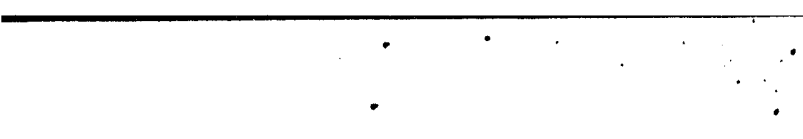

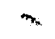
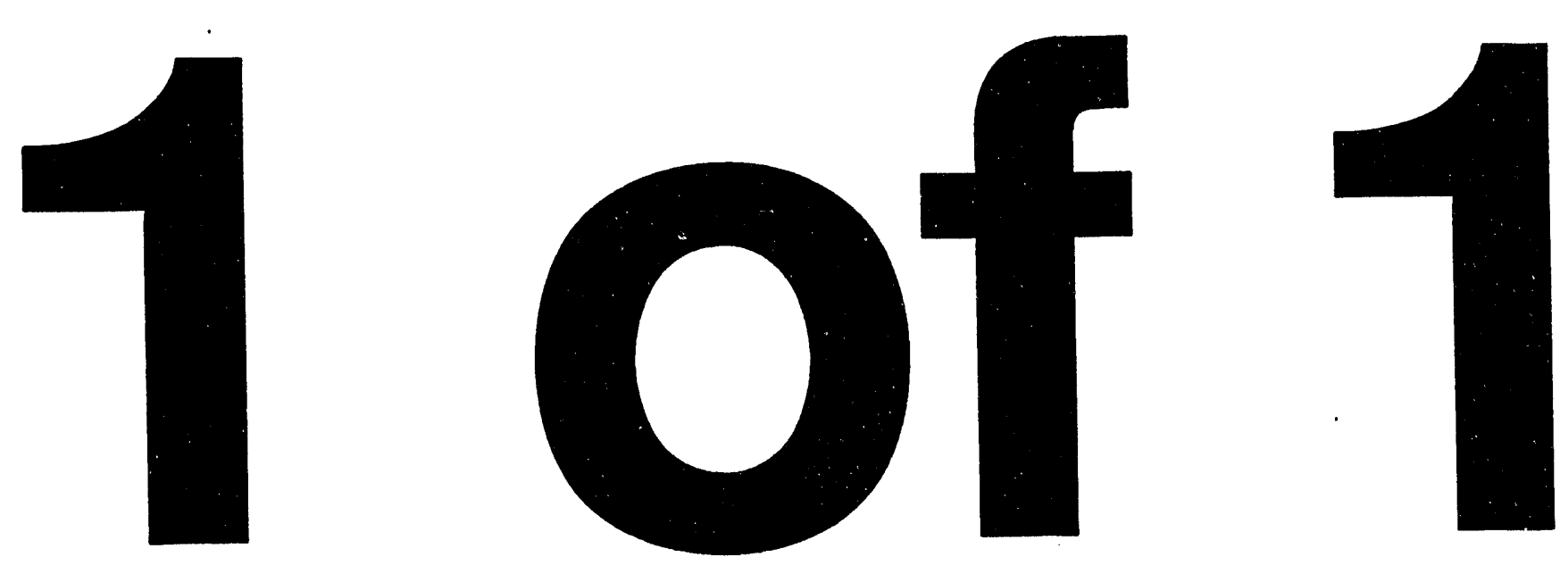


$$
\text { DOE/ER/40150-2.260 }
$$

\section{$9=$ CEBAF-TH-94-06 \\ The Continuous Electron Beam Accelerator Facility \\ Theory Group Preprint Series}

Additional copies are available from the authors.

The Southeastern Universities Research Association (SURA) operates the Continuous Electron Beam Accelerator Facility for the United States Department of Energy under contract DE-AC05-84ER40150

DISCLAIMER

This report was prepared as an account of work sponsored by the United States government. Neisber the United State nor the United States Department of Energy, nor any of their employes, makes any warransy, express or implied, or anumen any legal liability or responsibility for the accuracy, compleseness, or usefulness of any information, apparatus, product, or process disclosed, or represents that its use would not infringe privately owned rights. Reference herein to any specific commerical product. process, or service by trade name, mark, manufacturer, or otherwise. does not necesearily constitute or imply its endorsement. recommendation, or favoring by the United State government or any agency thereof. The views and opinion of authors expreased berein do not necessarily state or reflect those of the United Stales government or any agency thered. 


\title{
THE PROTON MAGNETIC FORM FACTOR IN A VECTOR MESON DOMINANCE MODEL
}

\author{
Robert A. Williams* and Siegfried Krewald ${ }^{\dagger}$ \\ Continuous Electron Beam Accelerator Facility \\ Newport News, Virginia 29606
}

\begin{abstract}
New precision data for the magnetic form factor of the proton in the time-like region is found to be well represented by a "dipole" vector meson dominance model for both space-like and time-like momentum transfers (for $\left|q^{2}\right|$ up to $\approx 15 \mathrm{GeV}^{2}$ ), if the first excited $\rho$-meson is taken into account. Our result gives a counter example to the previous claim that the new data is only well described by perturbative QCD.
\end{abstract}

Typeset using REVTEX

\footnotetext{
"Department of Physics, Hampton University, Hampton, VA 23668, USA

${ }^{\dagger}$ permanent address: Forschungszentrum Jülich, Institut für Kernphysik, D-52428 Jülich, Germany
} 
Recently, the proton magnetic form factor has been determined in the time-like region by measuring the exclusive cross section for proton-antiproton annihilation into the electronpositron final state, producing new precision data for inomentum transfers in the region $8.9 \mathrm{GeV}^{2} \leq q^{2} \leq 13 \mathrm{GeV}^{2}$ (Fermilab E-760) [1] and in the vicinity of the proton-antiproton threshold (LEAR PS170) [2]. Perturbative QCD (pQCD) calculations are usually compared with data for space-like momentum transfers larger than $Q^{2}=10 \mathrm{GeV}^{2}$ [3] [4]. The new Fermilab E-760 data confirm the predictions of perturbative QCD for the slope of magnetic form factor in the time-like region [1]. The vector meson dominance (VMD) model [5-10] , on the other hand, predicts a slope of the magnetic form factor of the proton at the proton-antiproton threshold which is about five times smaller than the the new Low Energy Antiproton Ring ( LEAR ) data [11]. Given the apparent failure of the vector meson dominance model and the success of $P Q C D$, the data have been used to derive a running coupling constant in the time-like region [1].

In this communication, we want to point out that a minimal modification of the vector meson dominance model produces a magnetic form factor which is in fair agreement with the data in both the space-like and time-like regions for momentum transfers up to $q^{2} \sim$ $15 \mathrm{GeV}^{2}$.

Ideally, one would like to predict form factors starting from the underlying quark dynamics. A quark model which incorporates the chiral symmetry of $Q C D$, the Nambu JonaLasinio model [12], has been developed quite successfully in recent years. Recent reviews can be found in Refs. [13] and [14]. Generalizations of the model are able to generate nucleon and meson form factors $[15,16]$ in good agreement with the experimental data for small space-like momentum transfers. At large space-like momentum transfers, however, a logarithmic divergence of the pion form factor has been found ( see Fig.7 of ref. [17] ). In the time-like region, applications of the Nambu Jona-Lasinio model are limited to relatively small excitation energies, because the model does not confine [14] , [18]. We therefore limit ourselves to a phenomenological analysis at present.

The nucleon electromagnetic form factors are typically parametrized by the dipole ansatz 


$$
F\left(q^{2}\right)=\left|F_{M}\left(q^{2}\right)\right|^{2}
$$

with $F_{M}\left(q^{3}\right)$ a monopole form factor

$$
F_{M}\left(q^{2}\right)=\frac{1}{1+\frac{q^{2}}{\Lambda_{M}^{2}}}
$$

The dipole parametrization is pure phenomenology since the square power of the monopole function is empirical and the effective energy scale $\Lambda_{M}$ is adjusted to give the best fit to the data without any theoretical constraint. The vector meson dominance model explains the energy scale $\Lambda_{M}$ by assuming that the photon couples to the nucleon via an intermediate vector meson. Here we show that from a meson-loop theory point of view, the dipole nature of the (iso-vector) nucleon form factors can be understood as the dominance of the (iso)vector meson resonances in the $\pi^{+} \pi^{-} \rightarrow p \bar{p}$ and $\rho \pi \pi \rightarrow p \bar{p}$ channels together with certain assumptions about how the off-shell vector mesons couple to the proton. We present clear phenomenological evidence that the proton magnetic form factor is well described by "dipole VMD", a parametrization which incorporates the physics of vector meson dominance, but with a dipole power-law which is derived from the assumption that the light meson loops which have relatively large coupling to the iso-vector meson resonances dominate the proton magnetic form factor. The resulting form factor takes on the form of a sum of dipole terms associated with the resonant $J=1, \pi \pi \rightarrow p \bar{p}$ and $\rho \pi \pi \rightarrow p \bar{p}$ partial wave scattering amplitudes (which we take to be dominated by the $\rho$ and $\rho^{\prime}=\rho(1600)$ respectively).

The most natural extension of the existing vector meson dominance models is to incorporate mesons with larger masses which may decay into electron-positron pairs. There are only two excited rho-mesons with masses below $2 \mathrm{GeV}$, the $\rho(1450)$ which decays mainly into four pions and the $\rho(1700)$ which dominantly decays into a rho and two pions. Before the analyses of [21] and [22], both rho-mesons were identified as one resonance, the $\rho(1600)$. Since we want to keep the number of free parameters minimal, we incorporate only one effective meson, which we call $\rho^{\prime}$ with a mass $m_{\rho^{\prime}}=1600 \mathrm{MeV}$. The influence of the narrow 
$\omega(1600)$ iso-scalar meson is thus effectively incorporated as well (although ambiguously). The $\omega(1600)$ decays mainly into a rho and a pion, but has also an electron-positron decay mode. We neglect the effect of the $\omega(1390)$, and we also do not Incorporate the $\phi$-mesons, assuming that the $\phi$-mesons are entirely made of strange quarks, and hence should be OZI suppressed (with no net strangeness in the nucleon). Another way to state our neglect of the iso-scalars is that we assume the magnetic proton form factor to be dominated by isovector currents. Furthermore, we assume that the coupling between the two-pion and the rho two-pion intermediate states is negligible.

We now define our dipole vector dominance model (see Fig.1):

$$
\begin{aligned}
G_{p}^{I V}\left(q^{2}\right) & \equiv F_{\pi}\left(q^{2}\right) \cdot \mathcal{M}_{\pi^{+} \pi^{-} \rightarrow p p}^{J=1}\left(q^{2}\right) \cdot \mathcal{I}_{\pi}\left(q^{2}\right) \\
& +F_{\rho}\left(q^{2}\right) \cdot \mathcal{M}_{\rho \pi \pi \rightarrow p \bar{p}}^{J=1}\left(q^{2}\right) \cdot \mathcal{I}_{\rho}\left(q^{2}\right),
\end{aligned}
$$

where $F_{\pi}\left(q^{2}\right)$, is the elastic charge form factor of the pion, $F_{\rho}\left(q^{2}\right)$ is related to the charge form factor of the rho meson (interpreting $\rho \pi \pi \sim \rho \rho), I_{\pi, \rho}\left(q^{2}\right)$ are calculable Feynman parameter integrals and $\mathcal{M}_{\pi^{+} \pi^{-} \rightarrow p p}^{J=1}\left(q^{2}\right), \mathcal{M}_{\rho \pi \pi \rightarrow p p}^{J=1}\left(q^{2}\right)$ are the $J=1$ (vector meson resonance dominated) partial wave amplitudes for the corresponding $\pi \pi$ and $\rho \pi \pi$ meson loops (see Fig.2). The form of Eqn.(3) is derived under the assumption that the "off-shell" pion form factor and hadronic scattering amplitudes are separable in the relative momentum variable and hence their respective $q^{2}$-dependence factorizes outside of the loop integral.

To be precise about our approximation for $G_{M}^{p}$, we now define a particular combination of the standard Dirac and Pauli form factors grouped according to isospin:

$$
\begin{aligned}
& G_{p}^{I V} \equiv \frac{1}{2}\left(F_{1}^{I V}+\kappa_{V} F_{2}^{I V}\right) \\
& G_{p}^{I S} \equiv \frac{1}{2}\left(F_{1}^{I S}+\kappa_{S} F_{2}^{I S}\right)
\end{aligned}
$$

where $\kappa_{V}$ and $\kappa_{S}$ are the ratios of tensor to vector coupling for isovector and isoscalar currents respectively (which determine the anomalous magnetic moment of the proton, $\kappa_{p} \equiv$ $\left.\frac{1}{2}\left(\kappa_{S}+\kappa_{V}\right)=1.793\right)$. In terms of these form factors, the magnetic proton form factor is given by 


$$
G_{M}^{p}=G_{p}^{I V}+G_{p}^{I S}
$$

Therefore, the approximation that $G_{M}^{p}$ is iso-vector dominated is formally equivalent to taking $G_{p}^{I V}>G_{p}^{I S}$, and hence

$$
G_{M}^{p}\left(q^{2}\right) \approx G_{p}^{I V}\left(q^{2}\right)
$$

If we view the graphs of Fig.1 as an equation for the $G_{p}^{I V}$ spectral function in dispersion theory, then we note that $\mathcal{M}_{\pi \pi \rightarrow p p}$ is complex in the time-like region with a phase that is conjugrate to $F_{\pi}$ and $\mathcal{M}_{\rho \pi \pi \rightarrow p p}$ has a phase conjugate to $F_{\rho}$ (analogous to the FSI theorem in elastic $\pi \pi$ scattering which ensures the spectral function is real). We can therefore write

$$
\begin{aligned}
& \mathcal{M}_{\pi \pi \rightarrow p \bar{p}} \equiv K_{\pi} F_{\pi}^{*}, \\
& \mathcal{M}_{\rho \pi \pi \rightarrow p \bar{p}} \equiv K_{\rho} F_{\rho}^{*} .
\end{aligned}
$$

In principle, the $K_{\pi}$ and $K_{\rho}$ factors are real functions of $q^{2}$ and the relative loop momentum, however we take them to be constants of proportionality. We can now re-write Eqn.(3) as

$$
\begin{aligned}
G_{p}^{I V}\left(q^{2}\right) & \equiv\left|\mathcal{M}_{\pi^{+}+\pi^{-} \rightarrow p \bar{p}}^{J=1}\left(q^{2}\right)\right|^{2} \cdot \frac{\mathcal{I}_{\pi}\left(q^{2}\right)}{K_{\pi}} \\
& +\left|\mathcal{M}_{\rho \pi \pi \rightarrow p \bar{p}}^{J=1}\left(q^{2}\right)\right|^{2} \cdot \frac{I_{\rho}\left(q^{2}\right)}{K_{\rho}} .
\end{aligned}
$$

We do not explicitly perform the Feynman parameter integrals to determine the $\mathcal{I}$-functions, however we note that one can obtain a wide range of phenomenological behavior depending on how the factorized off-shell form factors and amplitudes (i.e. the quantitative dependence on the relative loop momenta) are parametrized. We therefore assume that most of the $q^{2}$-dependence follows from the hadronic scattering amplitudes and treat the $\mathcal{I}_{\pi}$ and $\mathcal{I}_{p}$ functions as undetermined constants. The important point is that from Fig.2 and Eqn.(10) we can identify a 1 -parameter form for the phenomenological iso-vector proton form factor

$$
G_{p}^{I V}\left(q^{2}\right)=\frac{C}{\left(1-\frac{q^{2}}{M_{\rho}^{2}}\right)^{2}}+\frac{1-C}{\left(1-\frac{q^{2}}{M_{\alpha^{2}}^{2}}\right)^{2}},
$$

where $M_{\rho}=0.77 \mathrm{GeV}$ and $M_{\rho^{\prime}}=1.60 \mathrm{GeV}$ as previously discussed. We neglect the decay width effect in the time-like region since the widths are small compared to the nucleon mass, 
and also since both vector mesons lie below the $p \bar{p}$ threshold, there are no singularities in the physical region. There is one free parameter, i.e. " $\mathrm{C}$ ". In Fig.3, the magnetic form factor of the proton multiplied by $q^{4}$ and divided by the magnetic moment of the proton is displayed for both space-like and time-like momentum transfers. A value of $C=0.97$ has been chosen. The rescaled form factor predicted by perturbative QCD,

$$
\frac{q^{4} G_{P Q C D}^{p}\left(q^{2}\right)}{\mu_{p}}=\left(\frac{\alpha_{s}}{\ln \left(\frac{q^{2} \mid}{\Lambda_{Q C D}^{2}}\right)}\right)^{2}
$$

with $\alpha_{s}=5.25$ and $\Lambda_{Q C D}=0.2 \mathrm{GeV}$, is represented by the dotted line. Perturbative QCD does not predict the absolute magnitude of the cross sections, hence the normalization factor $\alpha_{s}=5.25$ is taken in order to achieve agreement with the data. We note that the new Fermilab data are larger by a factor of approximately 2 than the data at the corresponding space-like momentum transfers. Therefore, the form factors derived from perturbative QCD apparently need different normalizations in the space-like and time-like regions, as already noted in Ref. [1]. The dipole vector meson dominance model, on the other hand, generates an asymmetry between small space-like and time-like momentum transfers quite naturally because all meson poles are located at time-like momentum transfers. At momentum transfers much larger than the relevant meson masses, the asymmetry of the form factor vanishes in the dipole vector meson dominance model as well, of course, thus producing a behaviour similar to the one predicted by perturbative QCD. The LEAR data exhibit a slope near threshold which is much larger than the one expected by many vector meson dominance models [11]. The explicit consideration of the excited rho-meson in our dipole vector dominance model straightforwardly generates a form factor which depends more strongly on the momentum transfer just above the $p \bar{p}$ threshold. We find that the inclusion of the first excited rho-meson is sufficient to reproduce the slope of the experimental data near threshold.

There is one feature in the experimental data which cannot be explained by our ansatz Eqn.(3). The experimental form factor is nearly constant between $q^{2}=3.8 \mathrm{GeV}^{2}$ and $q^{2}=4.4 \mathrm{GeV}^{2}[1]$. Therefore, the rescaled form factor exhibits a strong $q^{2}$-dependence 
in the region under consideration, (since we chose to display the data multiplied by $q^{4}$, following Ref. [1] ). The peaking behavior in the rescaled form factor suggests that the broad, flat distribution seen in the un-scaled form factor corresponds to an additional resonance structure with a very large width. Since such a broad resonance can be incorporated into a phenomenological model only at the cost of additional free parameters, and since this effect is not large and does not change the important features of our model, we neglect it.

To summarize, we have shown that a generalization of the vector meson dominance model can describe the proton magnetic form factor up to momentum transfers of $q^{2} \approx$ $15 \mathrm{GeV}^{2}$. Both dipole vector meson dominance and perturbative QCD generate slopes of the form factors which agree with the data at large time-like momentum transfers. One has to conclude that the question: "which region of momentum transfer does a perturbative QCD treatment become necessary?" remains open.

\section{Acknowledgement}

The authors thank Nathan Isgur, Franz Gross and Warren Buck for their interest in this work. Discussions with C.-Y. (Alex) Pang concerning perturbative QCD are gratefully acknowledged. One of the authors ( S.K.) thanks Nathan Isgur and Franz Gross for their hospitality and financial support during his visit at CEBAF. 


\section{FIGURES}
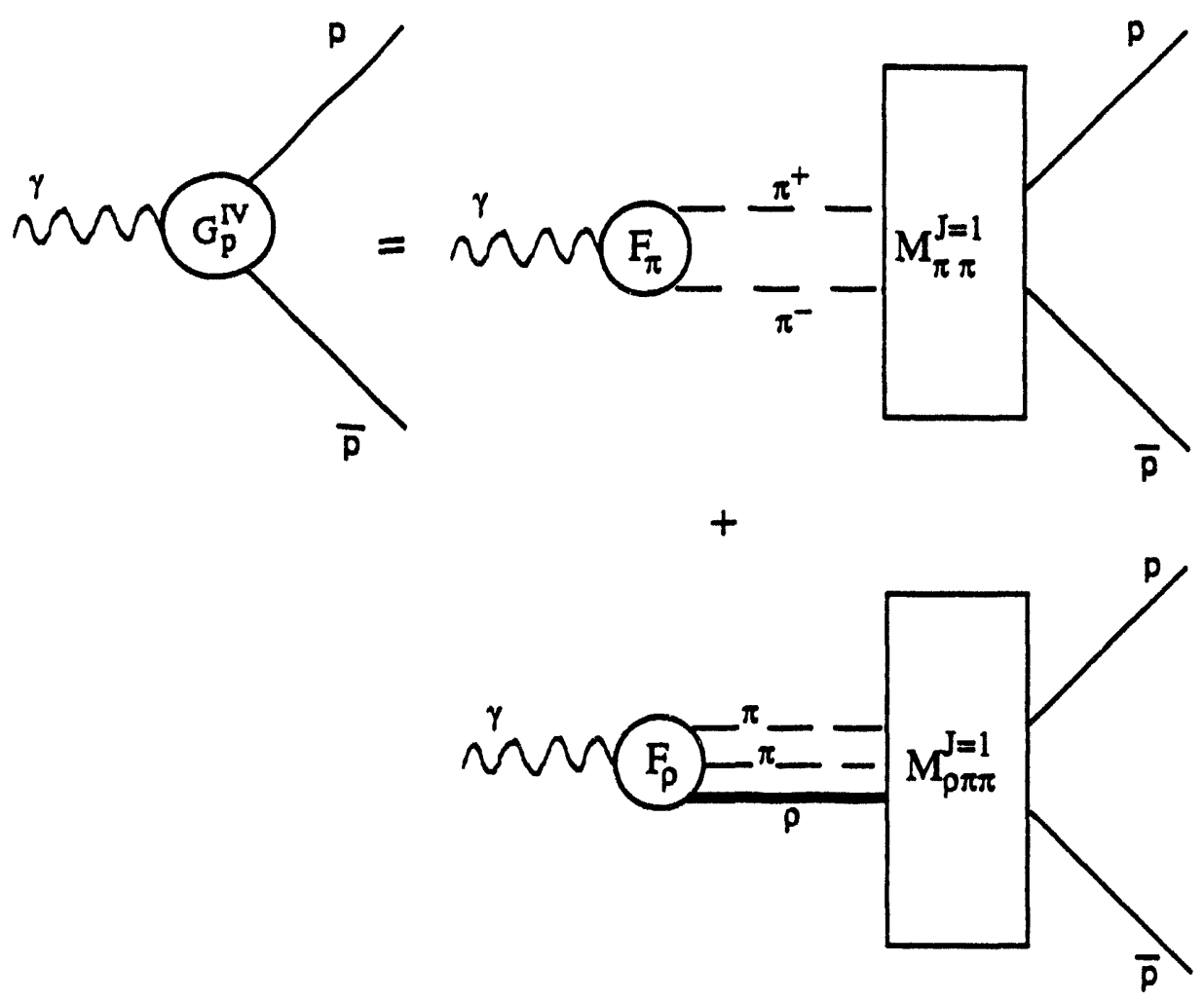

FIG. 1. We assume the iso-vector proton form factor $G_{p}^{I V}$ is dominated by re-scattering from light meson (i.e. $\pi \pi$ and $\pi \pi \rho$ ) intermediate states which couple strongly to $I=1$ vector mesons. 

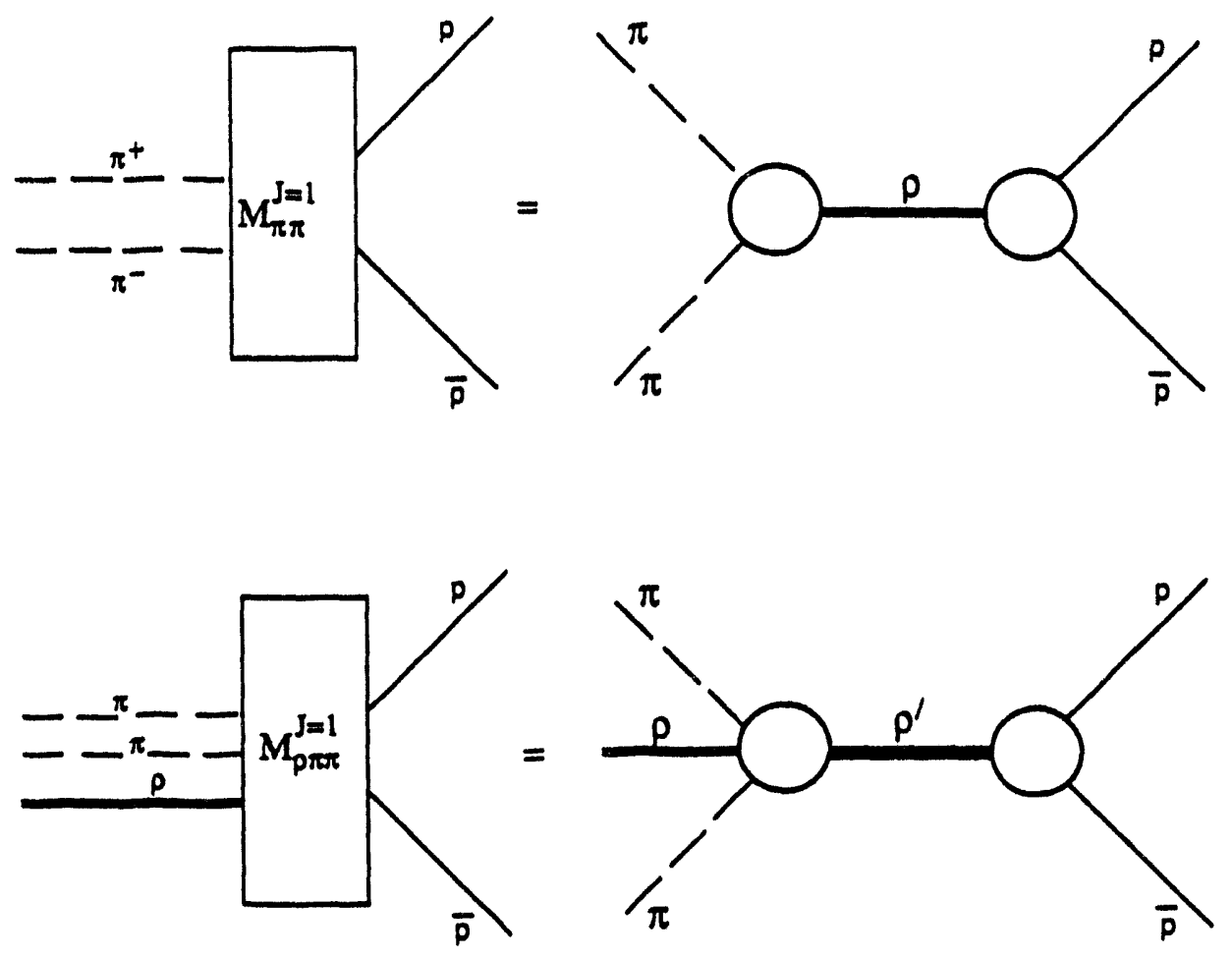

FIG. 2. The 2-pion intermediate state is assumed to re-scatter exclusively by the P-wave, rho resonance whereas the $J=1, \pi \pi \rho$ channel is dominated by the excited $\rho^{\prime}=\rho(1600)$ vector meson. We implicitly assume the hadronic couplings satisfy : $g_{\rho \pi \pi \rho}<<g_{\rho \pi \pi}$ and $g_{\rho^{\prime} \pi \pi}<<g_{\rho^{\prime} \pi \rho \rho}$. 


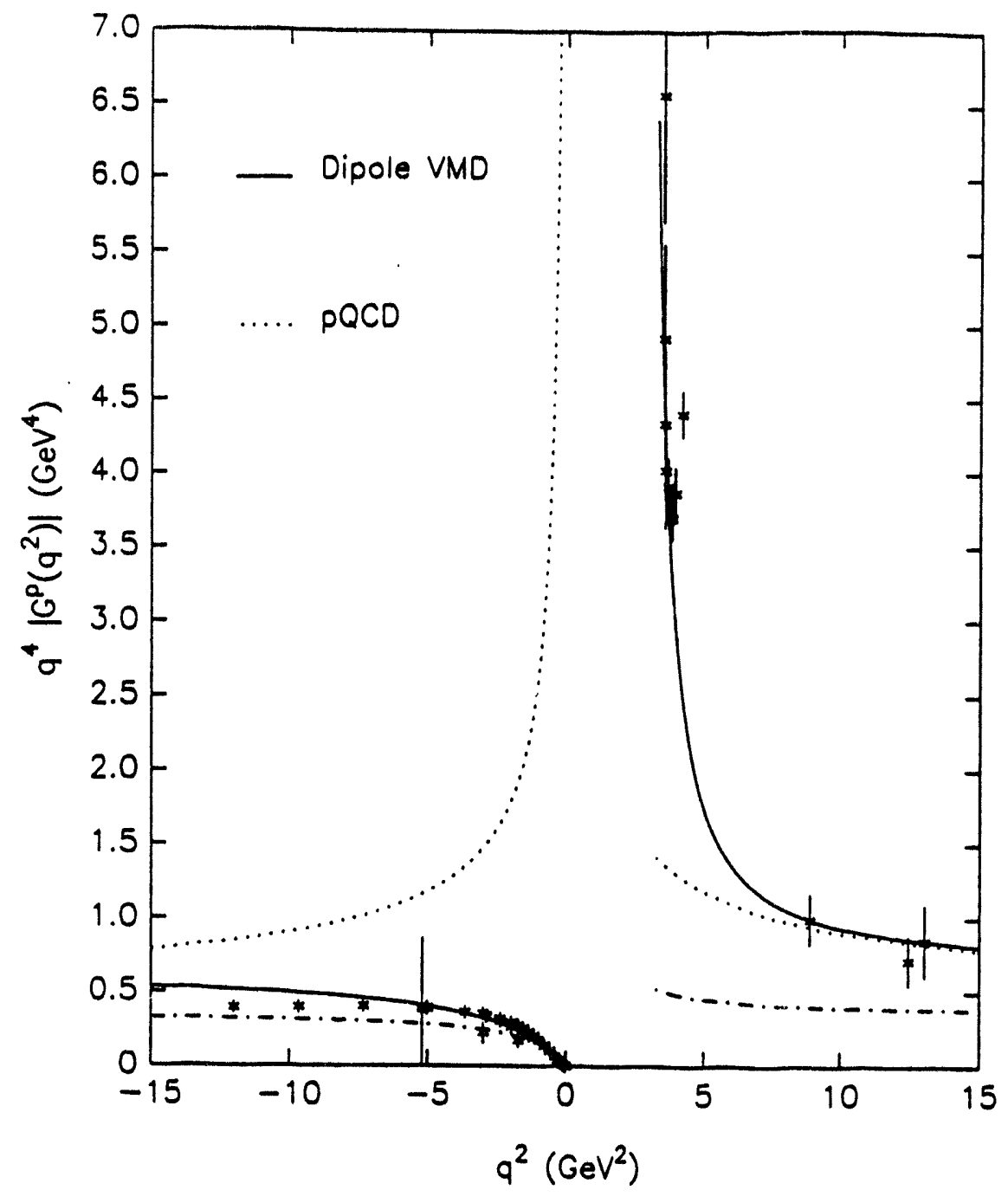

FIG. 3. The proton magnetic form factor, $G_{M}^{p}$, divided by the proton magnetic moment $\mu_{p}$ and scaled by $q^{4}$, is shown as a function of the square four-momentum transfer $q^{2}$. The generalized vector meson dominance model, Eqn.(11), is shown by the solid line whereas the dashed-dotted line shows the result without the excited $\rho^{\prime}$ resonance. The predictions of perturbative QCD are displayed by the dotted line (with normalization fixed in the time-like region). The data are from $\operatorname{Refs}[1,11,23,24]$. 


\section{REFERENCES}

[1] T.A.Armstrong et al., Phys.Rev.Lett.70,1212 (1991).

[2] G.Bardin et al., Phys.Lett.B255,149 (1991);B257,514 (1991).

[3] G.P.Lepage and S.J. Brodsky, Phys.Rev.Lett.43,545 (1979) ;Phys.Rev.D22,2157 (1980).

[4] N.G.Stefanis and M.Bergmann, Phys.Rev.D47,R3685(1993).

[5] M.Gell-Mann and F.Zachriasen, Phys.Rev.124,953 (1961).

[6] J.J.Sakurai, Currents and Mesons, Univ.of Chicago Press (1969).

[7] T.H. Bauer et al. Rev.Mod.Phys.50,161 (1978).

[8] T.Massam and A.Zichichi, Nuovo Cimento 43,1137 (1967).

[9] J.G.Koerner and M.Kuroda, Phys.Rev. D16,2165 (1977).

[10] S.Dubnicka and E.Etim, Nuovo Cimento A100,1 (1988).

[11] E.Luppi, Nucl.Phys. A558,165c (1993).

[12] Y.Nambu and G.Jona-Lasinio, Phys.Rev.122,345 (1961).

[13] S.P.Klevansky, Rev.Mod.Phys.64,649 (1992).

[14] U.Vogl and W.Weise, Prog.Part.Nucl.Phys. 27,195 (1991).

[15] P.Alberto et al., Phys.Lett.B208,75 (1988).

[16] V.Bernard and U.G.Meissner, Phys.Rev.Lett61,2296 (1988).

[17] V.Dmitransinovic, R.H.Lemmer, and R.Tegen, Comm. Nucl. and Part. Phys. 21, 71 (1993).

[18] S.Krewald, K.Nakayama, and J.Speth, Phys.Lett.B272,190 (1991).

[19] S.I.Bilenko, Y.M.Kazarinov, and L.I.Lapidus, Sov.Phys.JETP 33,247 (1971). 
[20] For inelastic channels with light meson intermediate states, the vector mesons determine both the behavior of the meson form factor and the rescattering into the $p \bar{p}$ final state.

[21] C.Erkal and M.G.Olsson, Z.Phys. C31,615 (1986).

[22] A.Donnachie and H.Mirzaie, Z.Phys. C33,407 (1986).

[23] G. Höhler et al., Nucl. Phys. B114, 505 (1976).

[24] G. Arnold et al., Phys. Rev. Lett. 57, 174 (1986). 
11
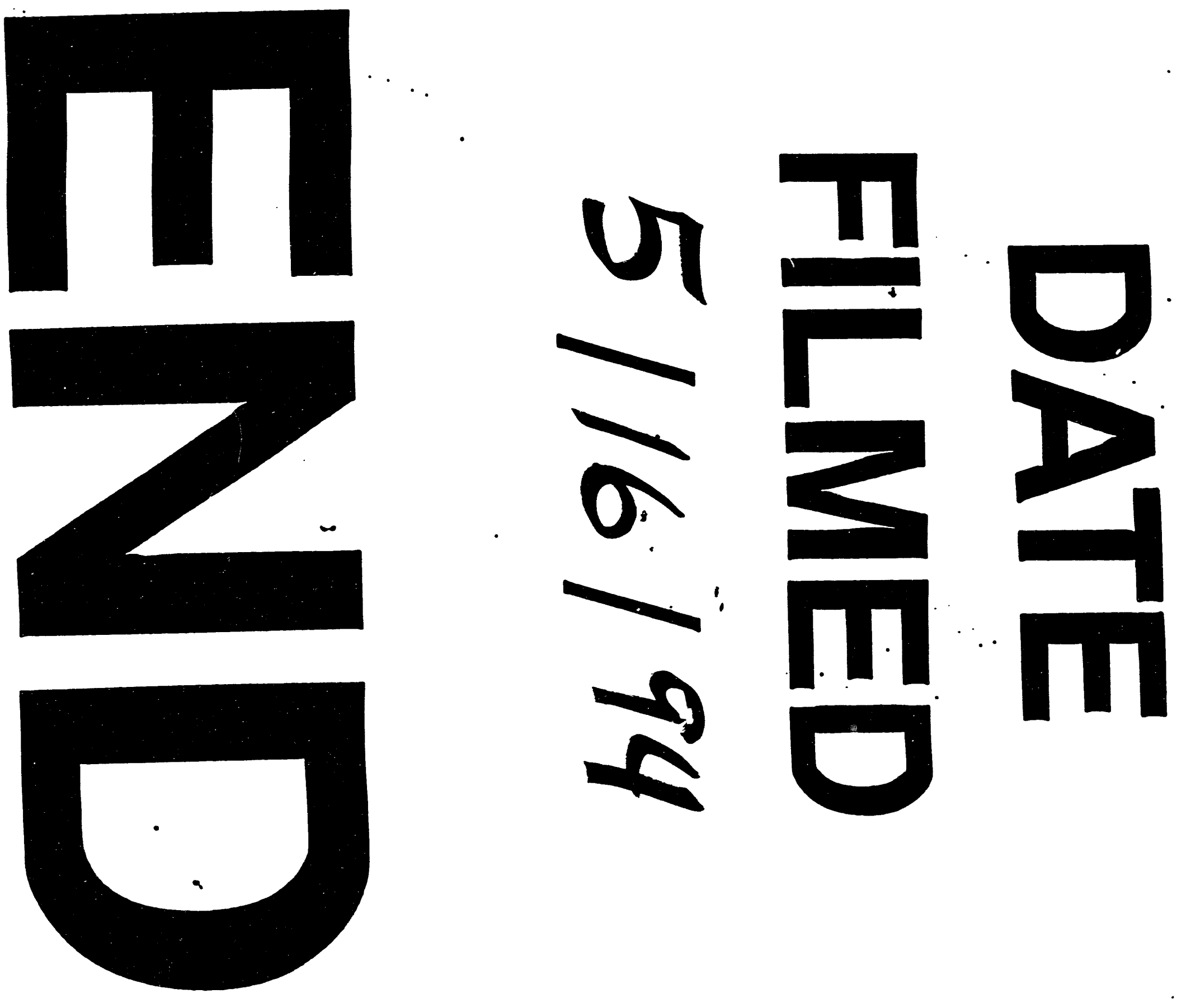


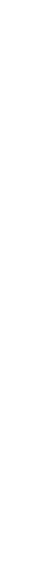

